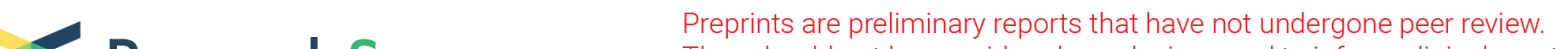

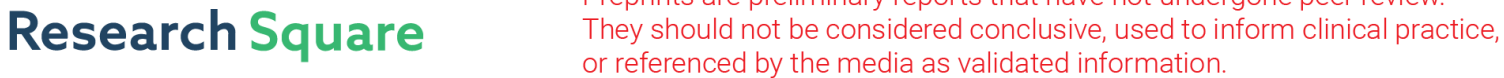

\section{Programmable Hybridization Assemble Nicked Displacement Amplification for Detecting Ricin Toxin}

\section{Yu Wang ( $\square$ wangyuyu9210@163.com )}

Tianjin Institute of Environmental and Operational Medicine https://orcid.org/0000-0001-7285-8004

\section{Yuan Peng}

Institute of Environmental and Operational Medicine

Jialei Bai

Institute of Environmental and Operational Medicine

Shuang Li

Institute of Environmental and Operational Medicine

\section{Dianpeng Han}

Institute of Environmental and Operational Medicine

\section{Shuyue Ren}

Institute of Environmental and Operational Medicine

\section{Kang Qin}

Institute of Environmental and Operational Medicine

\section{Sen Li}

Institute of Environmental and Operational Medicine

Huanying Zhou

Institute of Environmental and Operational Medicine

\section{Zhixian Gao}

Institute of Environmental and Operational Medicine

\section{Research}

Keywords: Hybridization assemble nicked displacement amplification, high sensitivity, ricin toxin, Programmable

Posted Date: September 24th, 2021

DOI: https://doi.org/10.21203/rs.3.rs-917396/v1

License: (c) (1) This work is licensed under a Creative Commons Attribution 4.0 International License. Read Full License 


\section{Abstract}

Isothermal amplification, such as hybridization chain reaction (HCR), is a simple and reliable method for detecting signal amplification. However, the hairpin in HCR will not fully participate in the reaction. And after the hairpin is opened, the distance between the fluorophore and the quencher does not change much. Therefore, the signal magnification is limited. Here, we designed a new isothermal amplification method named hybridization assemble nicked displacement amplification (HANDA), combining HCR and strand displacement amplification (SDA) ingeniously. HANDA first triggers HCR through the target sequence to form long double-strand DNA (dsDNA) with gaps. Then SDA is performed from the gap to obtain a large amount of single-stranded DNA (ssDNA), so as to achieve the purpose of double signal amplification. The base sequence of DNA hairpin had also been optimized. The best sequence design rule was found and had universal applicability. We have demonstrated that HANDA combined with DNA barcodes can be used for trace detection of ricin. This new isothermal amplification method provides an effective and universal platform for the trace detection of various toxic substances.

\section{Introduction}

Ricin toxin is a protein extracted from the seeds of castor, which is very resistant to acid and alkali [1, 2]. It is highly toxicity to the human body, and the toxicity is 6000 times that of cyanide. It can cause poisoning by breathing, intramuscular injection and skin contact [3-5]. Due to the simple preparation of ricin and the stable physical and chemical properties of the protein, it was added to the Schedule of the Chemical Weapons Convention (CWC) as the only protein toxin. It is also an important biological warfare agent clearly defined by the United Nations Convention on the Prohibition of Biological Weapons [6]. Ricin is extremely toxic and lethal in trace amounts $[7,8]$, so it is urgent to establish a highly sensitive detection method. Currently, enzyme-linked immunosorbent assay is the most widely used method for the detection of ricin [9-11]. Corresponding commercial detection kits have also been developed, but the sensitivity and detection range are limited [12-14]. In order to improve the detection sensitivity, our laboratory also tried to amplify the detection signal with isothermal amplification, and the detection sensitivity was improved to a certain extent [15]. However, we found that limited by the principle of HCR, the distance between the fluorophore and the quencher did not change much after the hairpin opened, so the degree of fluorescence signal recovery is limited.

Isothermal amplification is a technology that uses enzymes or spontaneous assembly of special DNA sequences in vitro to form a large amount of ssDNA/dsDNA at a constant temperature. It is an effective and convenient signal amplification method [16-18]. Common nucleic acid isothermal amplification methods include hybridization chain reaction (HCR) [19, 20], rolling circle amplification (RCA) $[21,22]$, strand displacement amplification (SDA) [23, 24], and Loop-mediated isothermal amplification (LAMP) $[25,26]$. According to previous laboratory experience, the primer design and reaction conditions of HCR and SDA are relatively simple. Moreover, the primers can be redesigned according to the different detection targets without affecting its amplification ability [27-29], which is an advantage that other amplification technologies cannot match. For example, Chen et al. designed a partial complementary 
sequence of aptamer and complementary DNA (CDNA) on the top of tetrahedral DNA to detect thrombin [30]. When thrombin was present, it would bind to the aptamer to release CDNA and cause HCR amplification. Shi et al. reported an exponential SDA for the detection of miRNA. The template of this method is specially designed so that the cleaved single-stranded sequence is the same as the primer sequence, so that the amount of template in each round of polymerization cycle is twice that of the previous round [31]. It seems that the two extended advantages can be combined to improve the abovementioned problem of limited signal amplification effects.

Herein, we ingeniously combined HCR and SDA and proposed a hybridization assemble nicked displacement amplification (HANDA). Hybridization of the target sequence with the hairpin triggered HCR to form a long double-stranded DNA with gaps. If the klenow fragment ( $\left.3^{\prime}-5^{\prime} \mathrm{exo}\right)$ enzyme (KF) combined to the gap, it would polymerize along 5'-3'. The HCR product could be disassembled to form multiple dsDNA. If there were nicking enzyme sites on the hairpin, each segment could be amplified by strand displacement to form a large amount of ssDNA. HANDA achieved the purpose of HCR amplifying the signal once and SDA amplifying the signal again. We also optimized the base sequence of the DNA hairpin, and found the best base sequence design rule with universal applicability. This new amplification method can not only achieve super amplification of the signal, but the base sequence can also be designed according to different targets. At the same time, we combined it with DNA barcodes (DNA barcodes-HANDA) to achieve ultra-sensitive detection of ricin. This method has great potential in the detection of trace amounts of pollutants.

\section{Materials And Methods}

The detailed procedure is provided in Supplementary Information (SI).

\section{Results And Discussion}

\section{Principle of Hybridization Assemble Nicked Displacement Amplification}

The principle of HANDA was shown in Fig. 1. The "head" of the hairpin L6SOH2 was designed with the recognition area of the Nt.BbvCl. Target $\mathrm{HO}$ (THO) bound to two hairpin probes, $\mathrm{L} 6 \mathrm{SOH} 1$ and $\mathrm{L} 6 \mathrm{SOH} 2$, and triggered HCR to form a long dsDNA with gaps. Then Klenow Fragment (3'-5'exo) polymerase (KF) were added to combined with the gap of the HCR product, and used one of the chains as a template for polymerization. When polymerized to the template chain gap, the long HCR fragment disintegrated, forming a double-stranded structure including $\mathrm{H} 1$ and $\mathrm{TH} 0$ sequence hybridization and $\mathrm{H} 1$ and $\mathrm{H} 2$ staggered hybridization, which contained a large number of $\mathrm{Nt.BbvCl}$ recognition sites. In the subsequent amplification, $\mathrm{Nt}$. BbvCl recognized and cleaved a single strand in the double strand to form a gap. KF combined with the gap and extended the DNA according to the template, and then replaced another ssDNA, which acted as a round of strand displacement amplification cycle. After several rounds of cycles, a large amount of ssDNA would be produced, which could hybridize with the fluorescent reporter probe to dissociate the DNA modified with the quenching group [32], resulting in the recovery of fluorescence 
intensity. If $\mathrm{TH} 0$ did not exist, $\mathrm{H} 1$ and $\mathrm{H} 2$ did not perform HCR. Moreover, the hairpin loop had a singlestranded structure, and $\mathrm{Nt}$. BbvCl could not recognize the cleavage, that is, non-specific amplification would not be triggered, ensuring a low background value. The base sequence of the non-cutting site can be arbitrarily designed according to the target, so this method can be used for the detection of various types of targets.

Agarose gel electrophoresis to verify the feasibility of HANDA

In the electrophoresis experiment, primers $\mathrm{L} 6 \mathrm{SOH} 1$ and $\mathrm{L} 6 \mathrm{SOH} 2$ were used to amplify hairpin probes, and the results were shown in Fig. 2A. Comparing lane 3 and lane 4, we could find that the band of lane 4 was significantly brighter, indicating that KF would polymerize along 5'-3' to complement the sticky end of L6SOH1 [33]. Moreover, no other miscellaneous strips appeared in the two lanes, which proved that the card issuing would not assemble spontaneously. Lane 5 showed bands of different lengths indicating that HCR occurred. Lane 6 was the system where KF was added after the HCR reaction, showing three bright bands: the bottom was the unreacted hairpin band; the middle band (about $70 \mathrm{bp}$ ) was the dsDNA formed by $\mathrm{KF}$ polymerization after the primer sequence hybridized with $\mathrm{L} 6 \mathrm{SOH} 1$; the upper band was a complete double-stranded structure formed by KF polymerization after dislocation hybridization of two $\mathrm{L} 6 \mathrm{SOH} 1$ and $\mathrm{L} 6 \mathrm{SOH} 2$. The length of it was about $90 \mathrm{bp}$, which was 1.5 times the hairpin after it was fully opened. Lane 7 showed a new short band. The added $\mathrm{Nt.BbvCl}$ would specifically recognize the site of the hairpin assembly product to cut the single strand, KF would bind to the gap and polymerize along the $5^{\prime}-3$ ' direction to replace the single strand. Lane 8 was the product formed by adding TH0, L6SOH1 and KF.

Amplification kinetics of HANDA

The two ends of the hairpin L6SOH2F were respectively labeled with fluorophore (6-FAM) and quencher (BHQ-1). When the hairpin was closed, 6-FAM and BHQ-1 were close to each other and the fluorescence was quenched. If the hairpin was opened, the fluorophore moved away from the quencher to restore the fluorescence. The dsDNA reporter probes were labeled with 6-FAM /BHQ-1. If TH0 was present, the dsDNA probe hybridized with the final amplification product, and 6-FAM would far away from the BHQ-1 to restore fluorescence. The amplification kinetics experiment measured the change of fluorescence intensity as the reaction progresses to infer the kinetics of amplification.

The result was shown in Fig. 2B. Section "a" was the HCR process in the presence of TH0. The sticky end of hairpin $\mathrm{L} 6 \mathrm{SOH} 1 \mathrm{~F}$ hybridized with TH0 so that the hairpin was opened to expose the recognition fragment of $\mathrm{L} 6 \mathrm{SOH} 1 \mathrm{~F}$, and then $\mathrm{L} 6 \mathrm{SOH} 2$ recognized and hybridized alternately. The progress of $\mathrm{HCR}$ made the fluorophore/quencher labeled at both ends away from each other to restore the fluorescence, which was specifically manifested as an increase in the fluorescence value of the "a". As the amplification progressing, the energy of HCR gradually entered a steady state, that is, the amplification basically stagnated (the fluorescence curve entered a plateau). The " $b$ " segment showed that the fluorescence value had further recovered. Because with the assistance of KF, the distance of some fluorophore/quencher pairs became farther (Fig. 2C). After adding Nt.BbvCl, the system would cut and 
polymerize the formed template. Hybridization of the amplified single strand with the reporter probe was achieved to continuously repeat the amplification process. More amplified fragments hybridized with the reporter probe to further increase the fluorescence value until the substrate was exhausted.

\section{Exploration of the sequence design rules of hairpin probe}

HANDA designed the nicking enzyme recognition sequence in the hairpin loop. Therefore, the number of bases in the hairpin loop and the size of the gap after the formation of dsDNA would affect the efficiency of HANDA. Gel electrophoresis and real-time fluorescence were used to explore the optimal number of bases in the hairpin loop. In this experiment, a total of 7 pairs of hairpins were designed: L6SOH1L6S0H2, L10S0H1-L10S0H2, L14S0H1-L14S0H2, L18S0H1-L18SOH2, L10S4H1-L10S4H2 L14S8H1L14S8H2, L18S12H1-L18S12H2. Among them, "L10" in the number "L10S4H1-L10S0H2" meant that the number of loop bases was 10; "S4" meant that the nicking enzyme recognition site was 4 bases away from the 5 'end.

Figure 3A showed the electrophoresis diagram of the assembled hairpins: L6SOH1-L6SOH2, L10SOH1L10SOH2, L14SOH1-L14SOH2, L18SOH1-L18SOH2. Among them, lanes 1-4 were hairpins mixed systems without THO. It could be seen that there was no amplified band except for the shallow band around $60 \mathrm{bp}$ in lane 3, indicating that the specificity of the hairpin was better. Lanes 5-8 were the HCR assembly system for hairpin after adding THO. Lanes 5 and 6 had amplified bands, indicating that L6SOH1L6S0H2, L10S0H1-L10S0H2 had stronger hybridization and amplification ability. Lanes 9-12 were added $\mathrm{KF}$ based on the previous period. It could be seen that two bands were produced at $70 \mathrm{bp}$ and $100 \mathrm{bp}$, and the band amplified by the original HCR basically disappeared. Comparing lanes 13-16 with lanes 9-12, there was a band at $20 \mathrm{bp}$, indicating that $\mathrm{Nt.BbvCl}$ could assist in amplifying a large amount of ssDNA. Moreover, the band in lane 13 was the brightest, proving that $\mathrm{L} 6 \mathrm{SOH} 1-\mathrm{L} 6 \mathrm{SOH} 2$ had the strongest hairpin amplification ability.

Real-time fluorescence quantitative PCR was used to monitor the fluorescence changes during the amplification process, and the ratio of each card signal value to the background value (signal-to-noise ratio) was determined when the amplification time was $120 \mathrm{~min}$ (Fig. 3B). The fluorescence values of L6S0H1-L6SOH2 reached a plateau first before $120 \mathrm{~min}$, and the signal-to-noise ratio was 12.0 (Fig. 3C). Compared with the signal-to-noise ratios of others, $\mathrm{L} 6 \mathrm{SOH} 1-\mathrm{L} 6 \mathrm{SOH} 2$ had the shortest amplification time, the highest signal-to-noise ratio and the strongest amplification ability. It might be that the two sticky ends of the hairpin were completely paired with the loop, making it easy to achieve a more stable state [34]. The results of this experiment were consistent with the conclusions of the electrophoresis experiment, so $\mathrm{L} 6 \mathrm{SOH} 1-\mathrm{L} 6 \mathrm{SOH} 2$ was used to for subsequent experiments.

Signal amplification capability of HANDA

The experiment used the same hairpin and THO concentration to compare the amplification ability (Fig. 4). The concentration of THO was quantified using conventional HCR amplification and the HANDA established in this experiment. The standard curve of conventional HCR was $y=1671.1 x-727.16$ (R2 = 
0.9924), the detection linear range was between $7.8125 \mathrm{nM}-1 \mu \mathrm{M}$, and the detection limit $(\mathrm{S} / \mathrm{N}=3)$ was $7.1 \mathrm{nM}$. The detection standard curve of HANDA was $y=1553.6 \mathrm{x}+2720.6(\mathrm{R} 2=0.9965)$, the detection linear range was $0.0305 \mathrm{nM}-250 \mathrm{nM}$, and the detection limit $(\mathrm{S} / \mathrm{N}=3)$ was $26.31 \mathrm{pM}$. The linear range of HANDA was one order of magnitude wider than that of the conventional HCR amplification detection method, and the sensitivity was also increased by 270 times, which proved that HANDA had super signal amplification capability, which could significantly improve detection sensitivity.

The principle and feasibility of ricin detection

The principle of ricin detection was shown in Fig. 5. The magnetic beads coupled with the ricin monoclonal antibody were sealed and combined with the ricin and the colloidal gold probe to form a sandwich structure. After removing the unreacted gold probe by magnetic separation, DTT was added. DTT destroyed the gold-sulfur bond formed by the sulfhydryl-labeled barcode DNA and the gold nanoparticles to free the barcode DNA bound to the gold probe. The supernatant, collected by magnetically separated, was mixed with $\mathrm{L} 6 \mathrm{SOH} 1$ and $\mathrm{L} 6 \mathrm{SOH} 2$. The barcoded DNA in the supernatant would trigger HCR to form a long chain structure with gaps that hybridized with $\mathrm{H} 1$ and $\mathrm{H} 2$. After adding KF and dNTP, KF could insert into the gap and then polymerize along the $5^{\prime}-3^{\prime}$ end with complementary single-stranded as a template, disintegrating the originally formed HCR long chain to form a doublestranded fragment containing barcode and $\mathrm{L} 6 \mathrm{SOH} 1$ hybridization, and the double-stranded fragments hybridized between $\mathrm{L} 6 \mathrm{SOH} 1$ and $\mathrm{L} 6 \mathrm{SOH} 2$, both of which were template of strand displacement amplification. Then the addition of $\mathrm{Nt.BbvCl}$ would recognize the sites on the templates to cut the single strands. KF inserted and then polymerized along the 5'-3' ends to replace the single strands, so that a large amount of ssDNA would be generated after continuous cycle amplification. They hybridized with the reporter probe to restore fluorescence. The fluorescence value was directly proportional to the concentration of the barcode DNA and ricin, which could detect the concentration of ricin with high sensitivity.

Three concentrations of ricin at $50 \mathrm{ng} / \mathrm{mL}, 5 \mathrm{ng} / \mathrm{mL}$, and $0 \mathrm{ng} / \mathrm{mL}$ were detected, and their fluorescence values showed a gradient downward trend, indicating that the presence of ricin could trigger subsequent HANDA (Fig. 6A). At the same time, the fluorescence intensity of the reporter probe and the negative value $(0 \mathrm{ng} / \mathrm{mL})$ basically overlap, indicating that when there was no target, the supernatant collected by magnetic separation did not contain barcode DNA, so the hairpin probe was stably present in the system.

Analytical performance of DNA barcodes-HANDA

After the detection system was optimized, the detection range and sensitivity of the method needed to be further investigated. The detection result was shown in Fig. 6B/C. As the concentration of ricin increased, the fluorescence value at $520 \mathrm{~nm}$ also gradually increased. Different concentrations of ricin samples were used as the abscissa, and the fluorescence value of the corresponding target minus the negative fluorescence value $(\triangle F)$ was used as the ordinate to establish a standard curve. In the range of 0.2441 $\mathrm{ng} / \mathrm{mL}-1000 \mathrm{ng} / \mathrm{mL}$, the detected fluorescence value had a good linear relationship with the 
concentration of ricin. The linear equation was $y=242.25 x+213.21\left(R^{2}=0.9926\right)$, and the detection limit $(\mathrm{S} / \mathrm{N}=3)$ was $85.11 \mathrm{pg} / \mathrm{mL}$.

Acacia toxin (Arbin), BSA, SEA, and IgG at a concentration of $20 \mathrm{ng} / \mathrm{mL}$ were selected as structural analogs for specificity experiments (Fig. 6D). Only ricin had an obvious response fluorescence value, and the fluorescence values of other targets were similar to the blank value. This was due to the good recognition specificity of monoclonal antibodies. In addition, this method had also been successfully applied to actual sample testing (Table S2).

\section{Conclusion}

Although HCR can continue until the hairpin is exhausted according to the principle, the hairpin does not fully participate in the reaction in actual experiments, and the amplification ability is affected by the "stem-loop base number ratio" of the hairpin [35], so the magnification is limited. In this experiment, HCR and SDA technology were combined to develop a strand displacement amplification technology based on hairpin assembly, which successfully overcomes the limitation of signal amplification. Meanwhile, we explored the optimal base sequence design rules for hairpin probes. Experiments confirmed that the number of bases in the hairpin loop iwas 6 and the amplification efficiency and ability were higher when the restriction site overlaps with the HCR assembly breakpoint. Compared with the conventional HCR, this amplification method exhibited a 270-fold increase in sensitivity and a linear range that had been extended by an order of magnitude. The sequence of the non-cutting site can be arbitrarily designed according to the target, so this method has a certain versatility. Combining HANDA with DNA barcode to detect ricin, the results showed that within the range of $0.2441 \mathrm{ng} / \mathrm{mL}-1000 \mathrm{ng} / \mathrm{mL}$, the detected fluorescence value had a good linear relationship with the concentration of ricin. Moreover, this method can be successfully used for the detection of ricin in food.

\section{Declarations}

\section{Supplementary Information}

Supplementary data associated with this article can be found, in the online version, at https://

\section{Acknowledgments}

The authors thank the Natural Science Foundation of Tianjin City (Grant No. 18YFZCNC01260) for and the National Key Research and Development Program of China (Grant No. 19SWAQ21) for funding this research project.

\section{Author Contributions}

YW conceived of the project and contributed to the conception, fabrication and writing of the manuscript. ZXG supervised the project. YP and JLB contributed to the principle design. SL and DPH contributed to 
the detection experiments. SYR contributed to figure processing. KQ contributed to the manuscript modification. SL and HYZ contributed to data analysis. The manuscript was written through contributions of all authors. All authors have given approval to the final version of the manuscript.

\section{Availability of data and materials}

All data generated or analyzed during this study are included in the article and additional file.

\section{Ethics approval and consent to participate}

Not applicable.

\section{Consent for publication}

All authors have provided consent for the manuscript to be published.

\section{Competing interests}

The authors declare no competing fnancial interest

\section{Author details}

a Tianjin Key Laboratory of Risk Assessment and Control Technology for Environment and Food Safety, Tianjin Institute of Environmental and Operational Medicine, Tianjin 300050, P.R. China.

\section{References}

1. Endo Y, Tsurugi K. RNA N-glycosidase activity of ricin A-chain. Mechanism of action of the toxic lectin ricin on eukaryotic ribosomes. J Biol Chem. 1987;262:8128-30.

2. Stine R, Pishko MV, Schengrund CL. Comparison of glycosphingolipids and antibodies as receptor molecules for ricin detection. Anal Chem. 2005;77:2882-8.

3. Olsnes S, Pihl A. Isolation and properties of abrin: a toxic protein inhibiting protein synthesis. Evidence for different biological functions of its two constituent-peptide chains. Eur J Biochem. 1973;35:179-85.

4. Rutenber E, Robertus JD. Structure of ricin B-chain at 2.5 A resolution. Proteins. 1991;10:260-9.

5. Olsnes S. The history of ricin, abrin and related toxins. Toxicon. 2004;44:361-70.

6. Atlas RM. Bioterriorism: from threat to reality. Annu Rev Microbiol. 2002;56:167-85.

7. Soliman SSM, Baldin C, Gu Y, Singh S, Gebremariam T, Swidergall M, Alqarihi A, Youssef EG, Alkhazraji S, Pikoulas A, et al: Mucoricin is a ricin-like toxin that is critical for the pathogenesis of mucormycosis. Nat Microbiol 2021.

8. Zhao X, Li H, Li J, Liu K, Wang B, Wang Y, Li X, Zhong W. Novel small molecule retrograde transport blocker confers post-exposure protection against ricin intoxication. Acta Pharm Sin B. 2020;10:498- 
511.

9. Heller NC, Garrett AM, Merkley ED, Cendrowski SR, Melville AM, Arce JS, Jenson SC, Wahl KL, Jarman $\mathrm{KH}$. Probabilistic Limit of Detection for Ricin Identification Using a Shotgun Proteomics Assay. Anal Chem. 2019;91:12399-406.

10. Sun J, Zhang X, Li T, Xie J, Shao B, Xue D, Tang X, Li H, Liu Y. Ultrasensitive On-Site Detection of Biological Active Ricin in Complex Food Matrices Based on Immunomagnetic Enrichment and Fluorescence Switch-On Nanoprobe. Anal Chem. 2019;91:6454-61.

11. Ye QC, Men C, Li YF, Liu JJ, Huang CZ, Zhen SJ. Catalytic hairpin assembly mediated liposomeencoded magnetic beads for signal amplification of peroxide test strip based point-of-care testing of ricin. Chem Commun (Camb). 2020;56:14091-4.

12. Garber EA, Thole J. Application of Microwave Irradiation and Heat to Improve Gliadin Detection and Ricin ELISA Throughput with Food Samples. Toxins (Basel). 2015;7:2135-44.

13. Chileshe J, Goosen WJ, Buss PE, van Helden PD, Warren R, Parsons SDC, Miller MA. A commercial ELISA for detection of interferon gamma in white rhinoceros. J Vet Diagn Invest. 2019;31:531-6.

14. Battaglia F, Meucci V, Tognetti R, Bonelli F, Sgorbini M, Lubas G, Pretti C, Intorre L. Procalcitonin Detection in Veterinary Species: Investigation of Commercial ELISA Kits. Animals (Basel). 2020;10:1511.

15. Yuan S, Huo B, Zhang M, Wang Y, Bai J, Peng Y, Ning B, Chen P, Gao Z. Dual signal amplification strategy for detection of ricin toxin through bio-barcode assay combined with hybridization chain reaction. Chinese Journal of Analycial Chemistry. 2019;47:156-63.

16. Zhao Y, Chen F, Li Q, Wang L, Fan C. Isothermal Amplification of Nucleic Acids. Chem Rev. 2015;115:12491-545.

17. Janik M, Hamidi SV, Koba M, Perreault J, Walsh R, Bock WJ, Smietana M. Real-time isothermal DNA amplification monitoring in picoliter volumes using an optical fiber sensor. Lab Chip. 2021;21:397404.

18. Wang R, Qian C, Pang Y, Li M, Yang Y, Ma H, Zhao M, Qian F, Yu H, Liu Z, et al. opvCRISPR: One-pot visual RT-LAMP-CRISPR platform for SARS-cov-2 detection. Biosens Bioelectron. 2021;172:112766.

19. Dirks RM, Pierce NA. Triggered amplification by hybridization chain reaction. Proc Natl Acad Sci U S A. 2004;101:15275-8.

20. Zeng Z, Zhou R, Sun R, Zhang X, Cheng Z, Chen C, Zhu Q. Nonlinear hybridization chain reactionbased functional DNA nanostructure assembly for biosensing, bioimaging applications. Biosens Bioelectron. 2020;173:112814.

21. Mohsen MG, Kool ET. The Discovery of Rolling Circle Amplification and Rolling Circle Transcription. Acc Chem Res. 2016;49:2540-50.

22. Xu L, Duan J, Chen J, Ding S, Cheng W. Recent advances in rolling circle amplification-based biosensing strategies-A review. Anal Chim Acta. 2021;1148:238187. 
23. Zhou W, Hu L, Ying L, Zhao Z, Chu PK, Yu XF. A CRISPR-Cas9-triggered strand displacement amplification method for ultrasensitive DNA detection. Nat Commun. 2018;9:5012.

24. Zhang Y, Wang W, Lin Z, Liu B, Zhou X. Dual-output toehold-mediated strand displacement amplification for sensitive homogeneous electrochemical detection of specie-specific DNA sequences for species identification. Biosens Bioelectron. 2020;161:112256.

25. Nishino I, Fu J, Tanji K, Yamada T, Shimojo S, Koori T, Mora M, Riggs JE, Oh SJ, Koga Y, et al. Primary LAMP-2 deficiency causes X-linked vacuolar cardiomyopathy and myopathy (Danon disease). Nature. 2000;406:906-10.

26. Dao Thi VL, Herbst K, Boerner K, Meurer M, Kremer LP, Kirrmaier D, Freistaedter A, Papagiannidis D, Galmozzi C, Stanifer ML, et al: A colorimetric RT-LAMP assay and LAMP-sequencing for detecting SARS-CoV-2 RNA in clinical samples. Sci Trans/ Med 2020, 12:eabc7075.

27. Reid MS, Le XC, Zhang H. Exponential Isothermal Amplification of Nucleic Acids and Assays for Proteins, Cells, Small Molecules, and Enzyme Activities: An EXPAR Example. Angew Chem Int Ed Engl. 2018;57:11856-66.

28. Gines G, Menezes R, Xiao W, Rondelez Y, Taly V. Emerging isothermal amplification technologies for microRNA biosensing: Applications to liquid biopsies. Mol Aspects Med. 2020;72:100832.

29. Qi H, Yue S, Bi S, Ding C, Song W. Isothermal exponential amplification techniques: From basic principles to applications in electrochemical biosensors. Biosens Bioelectron. 2018;110:207-17.

30. Chen YX, Huang KJ, He LL, Wang YH. Tetrahedral DNA probe coupling with hybridization chain reaction for competitive thrombin aptasensor. Biosens Bioelectron. 2018;100:274-81.

31. Shi C, Liu Q, Ma C, Zhong W. Exponential strand-displacement amplification for detection of microRNAs. Anal Chem. 2014;86:336-9.

32. Joyce CM. How DNA travels between the separate polymerase and 3'-5'-exonuclease sites of DNA polymerase I (Klenow fragment). J Biol Chem. 1989;264:10858-66.

33. Beese LS, Derbyshire V, Steitz TA. Structure of DNA polymerase I Klenow fragment bound to duplex DNA. Science. 1993;260:352-5.

34. Zhang DY, Winfree E. Control of DNA strand displacement kinetics using toehold exchange. J Am Chem Soc. 2009;131:17303-14.

35. Choi HM, Beck VA, Pierce NA. Next-generation in situ hybridization chain reaction: higher gain, lower cost, greater durability. ACS Nano. 2014;8:4284-94.

\section{Figures}




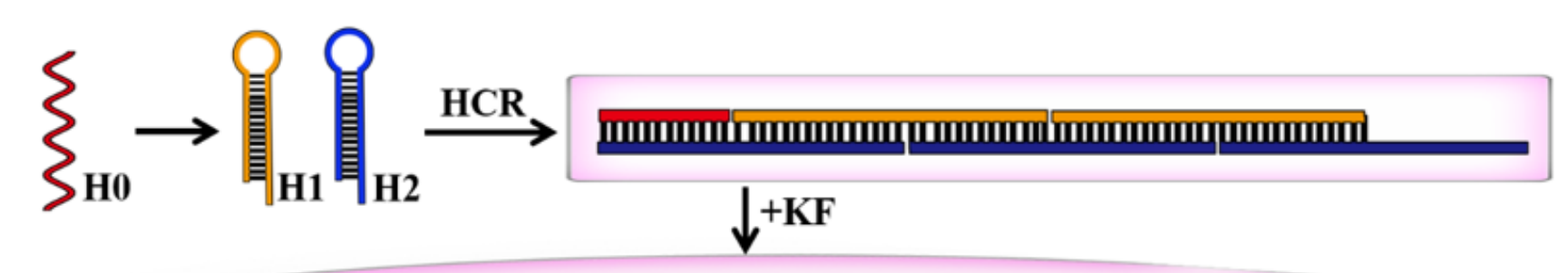
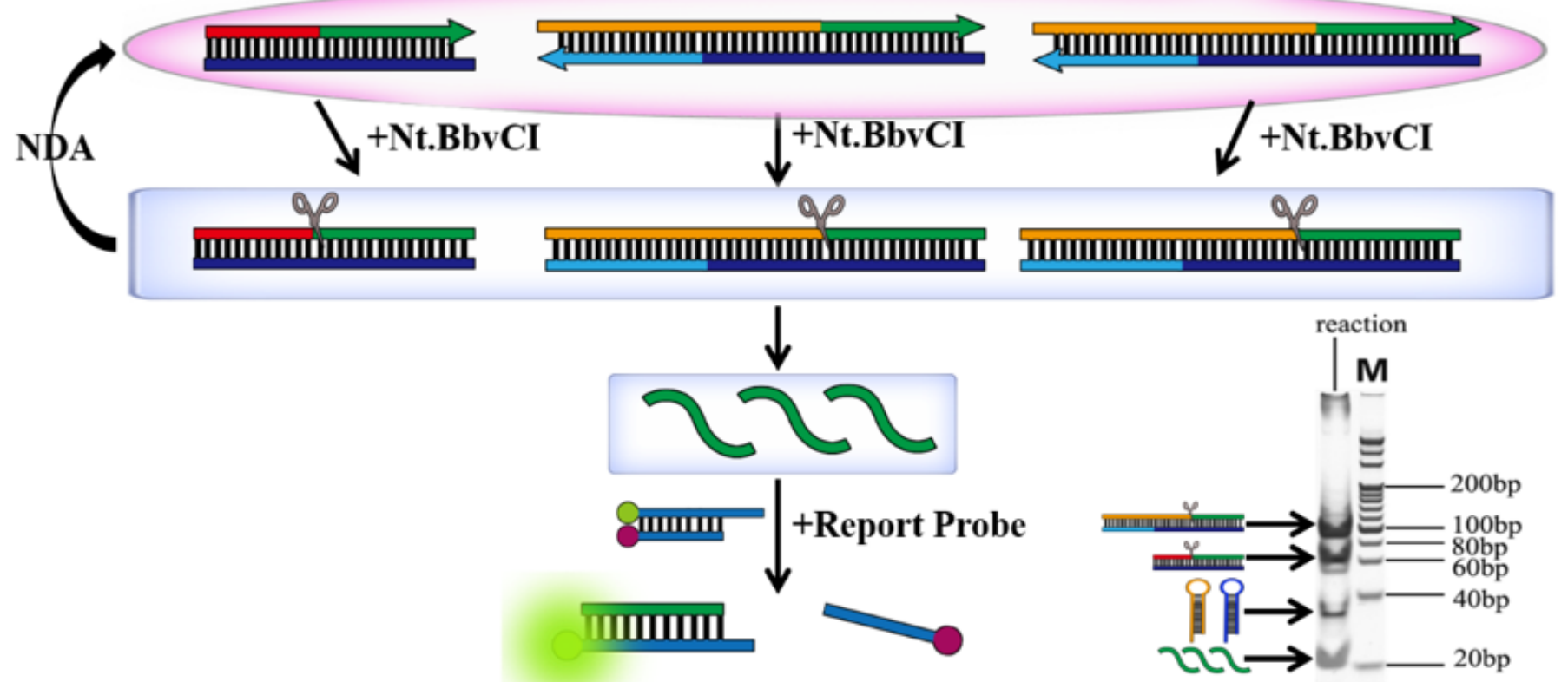

Figure 1

The schematic showing the principle of hairpin assembly nicked displacement amplification.
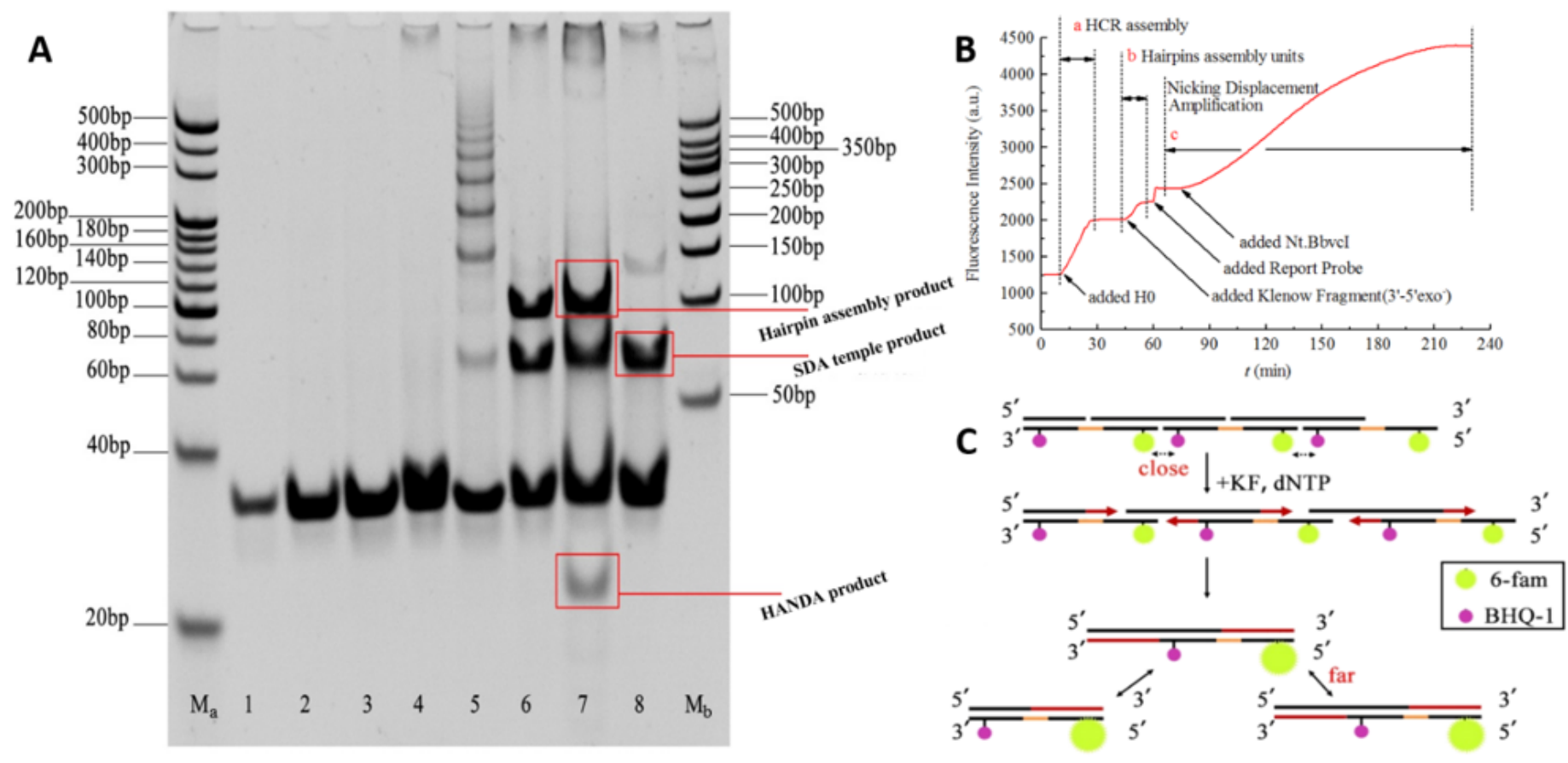

Figure 2 
(A) Feasibility experiment of hairpin assembly nicked displacement amplification (Ma: 20bp Marker, Mb:50bp ladder, 1: L6SOH1, 2: L6SOH2, 3: L6SOH1+L6SOH2, 4: L6SOH1+L6SOH2+ KF+Nt.BbvCl, 5: L6SOH1+L6SOH2+TH0, 6: L6SOH1+L6SOH2+TH0+KF, 7: L6SOH1+L6SOH2+ TH0+KF+Nt.BbvCl, 8: $\mathrm{L} 6 \mathrm{SOH} 1+\mathrm{TH} 0+\mathrm{KF})$; (B) fluorescence value versus time curve by HANDA; (C) schematic of KF polymerase was added fluorescence recovery after HCR assembly.
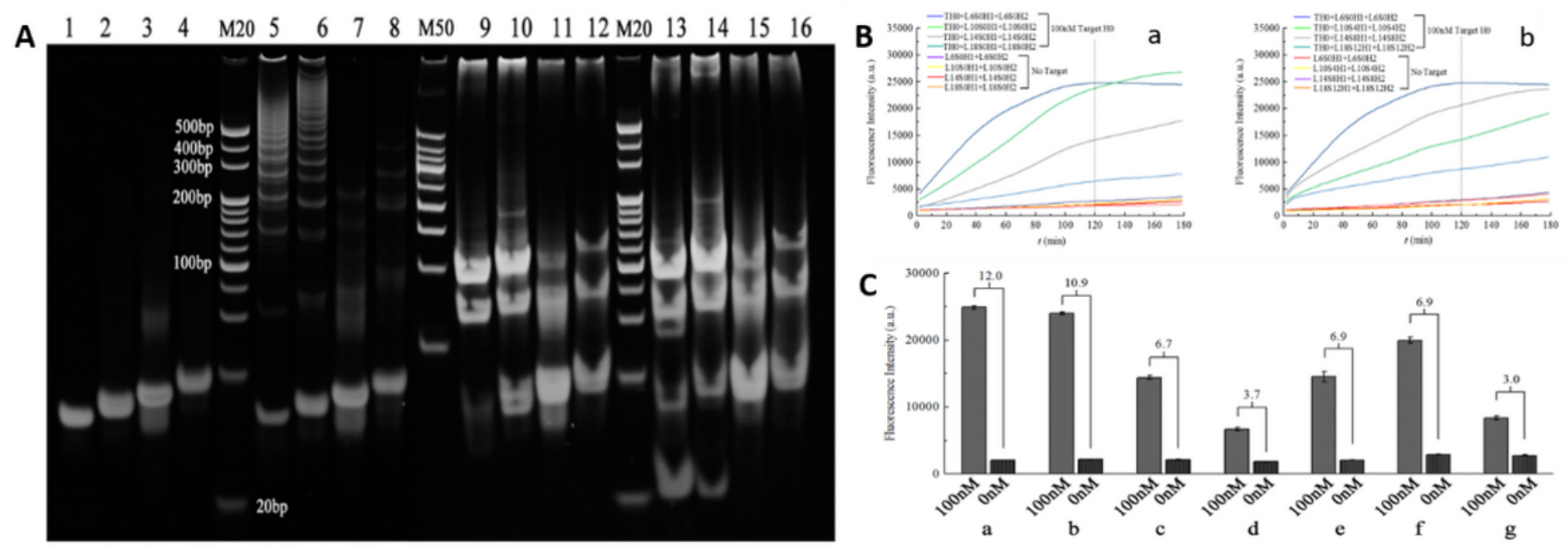

\section{Figure 3}

(A) Electrophoresis with different loops of base pairs (M20: 20bp Marker, M50: 50bp Ladder, 1: L6SOH1+L6SOH2, 2: L10SOH1+L10S0H2, 3: L14SOH1+L14SOH2, 4: L18SOH1+ L18SOH2, 5: L6SOH1 +L6SOH2+TH0, 6: L10SOH1+L10SOH2+TH0, 7: L14SOH1+L14SOH2+ TH0, 8: L18SOH1+L18SOH2+TH0, 9: L6SOH1+L6SOH2+TH0+KF, 10: L10S0H1+L10SOH2+ TH0+KF, 11: L14SOH1+L14SOH2+TH0+KF, 12: L18SOH1+L18SOH2+TH0+KF, 13: L6SOH1+ L6SOH2+TH0+KF+Nt.BbvCl, 14: L10SOH1+L10SOH2+TH0+KF+Nt.BbvCl, 15: L14SOH1+ L14SOH2+THO+KF+Nt.BbvCl, 16 : $\mathrm{L} 18 \mathrm{SOH} 1+\mathrm{L} 18 \mathrm{SOH} 2+\mathrm{TH} 0+\mathrm{KF}+\mathrm{Nt} . \mathrm{BbvCl}$ ); (B) fluorescence value versus time curve with different length loops of assembly amplification hairpins; (C) signal to noise ratio with different length loops of hairpins. 


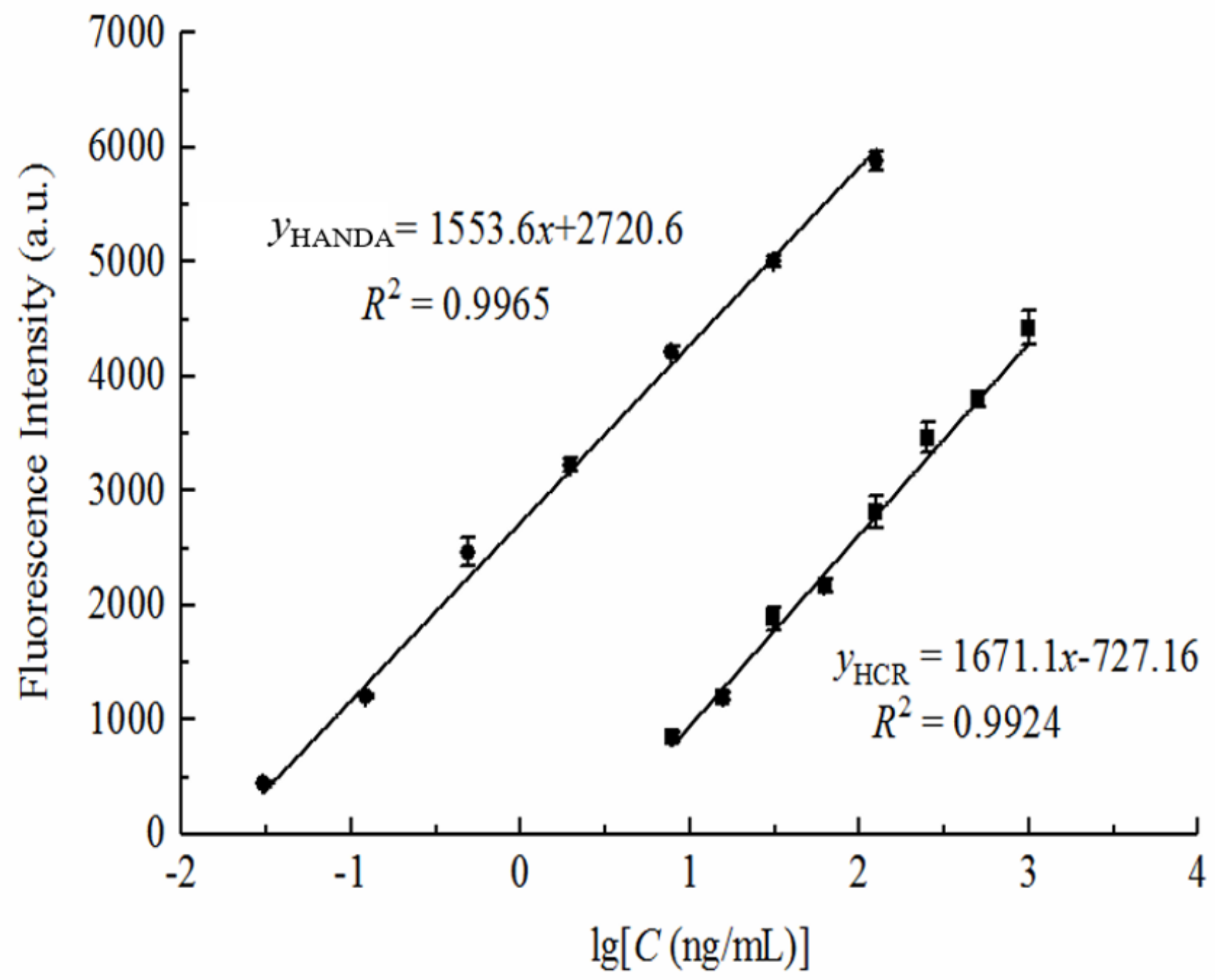

Figure 4

Standard curve of detect targets by this method and conventional HCR method. 


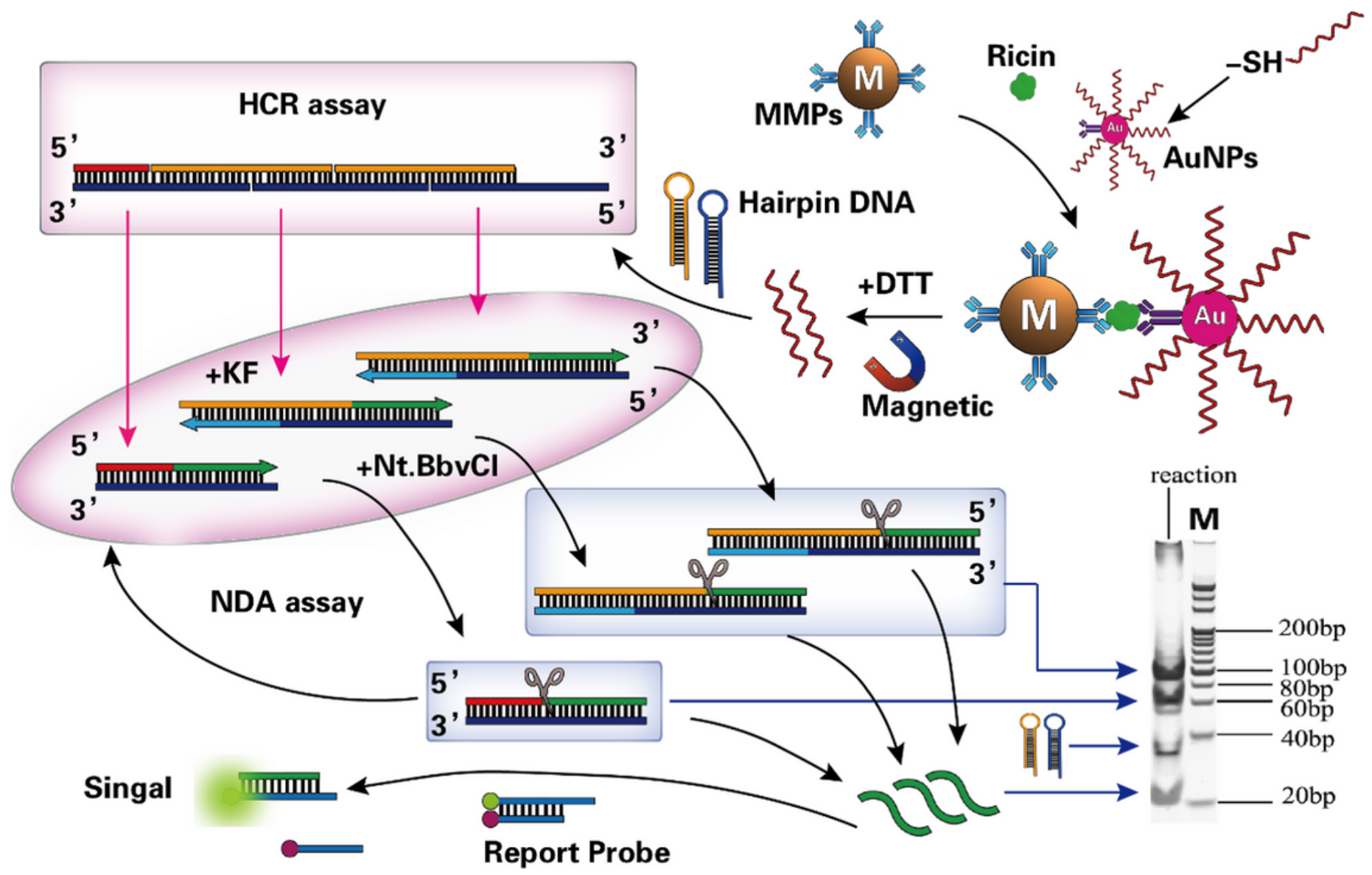

Figure 5

The principle of Bio-barcode combined with hairpin assembly nicked displacement amplification to detect ricin. 

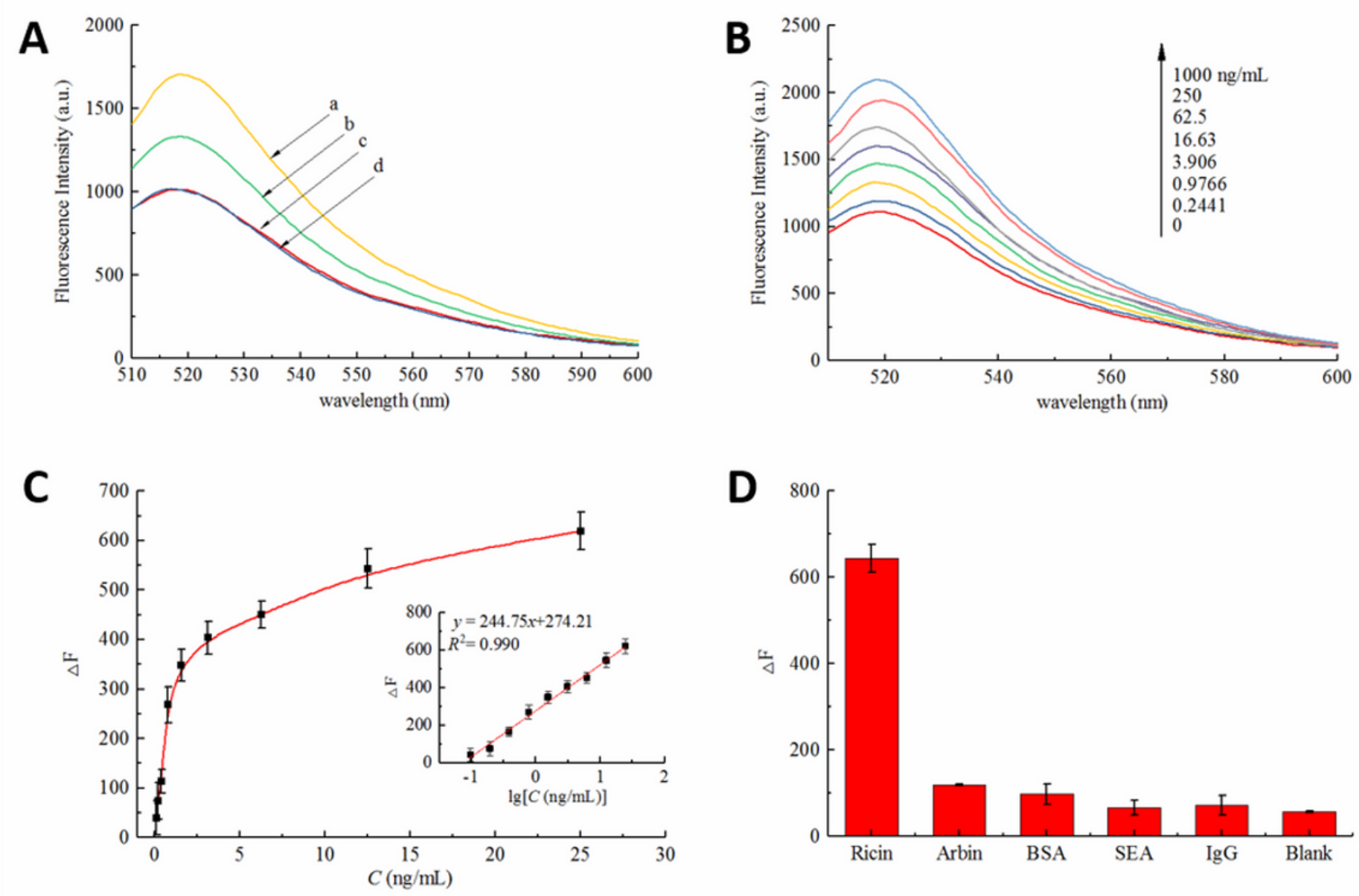

Figure 6

(A) Feasibility experiment of this method: concentration of ricin is $50 \mathrm{ng} / \mathrm{mL}$ (a), $5 \mathrm{ng} / \mathrm{mL}$ (b) and 0 $\mathrm{ng} / \mathrm{mL}$ (c); (B) fluorescence spectrum curves measured by this method for different concentrations of ricin; (C) standard curve for detecting different concentrations of ricin; (D) specificity test of this method.

\section{Supplementary Files}

This is a list of supplementary files associated with this preprint. Click to download.

- GraphicalAbstracts.docx

- SIHANDA.docx 